\title{
Genome-wide identification, characterization and expression analysis of the non-specific lipid transfer proteins in potato
}

Guojun Li ${ }^{1}$, Menglu Hou' ${ }^{1}$ Yaxue Liu ${ }^{2}$, Yue Pei ${ }^{1}$, Minghui Ye ${ }^{1}$, Yao Zhou ${ }^{1}$, Chenxi Huang ${ }^{1}$, Yaqi Zhao and Haoli $\mathrm{Ma}^{1^{*}}$ (D)

\begin{abstract}
Background: Plant non-specific lipid transfer proteins (nsLTPS) are small, basic proteins that are abundant in higher plants. They have been reported to play an important role in various plant physiological processes, such as lipid transfer, signal transduction, and pathogen defense. To date, a comprehensive analysis of the potato nsLTP gene family is still lacking after the completion of potato (Solanum tuberosum L.) genome sequencing. A genome-wide characterization, classification and expression analysis of the StnsLTP gene family was performed in this study.

Results: In this study, a total of 83 nsLTP genes were identified and categorized into eight types based on Boutrot's method. Multiple characteristics of these genes, including phylogeny, gene structures, conserved motifs, protein domains, chromosome locations, and cis-elements in the promoter sequences, were analyzed. The chromosome distribution and the collinearity analyses suggested that the expansion of the StnsLTP gene family was greatly enhanced by the tandem duplications. Ka/Ks analysis showed that 47 pairs of duplicated genes tended to undergo purifying selection during evolution. Moreover, the expression of StnsLTP genes in various tissues was analyzed by using RNA-seq data and verified by quantitative real-time PCR, revealing that the StnsLTP genes were mainly expressed in younger tissues. These results indicated that StnsLTPs may played significant and functionally varied roles in the development of different tissues.
\end{abstract}

Conclusion: In this study, we comprehensively analyzed nsLTPs in potato, providing valuable information to better understand the functions of StnsLTPs in different tissues and pathways, especially in response to abiotic stress.

Keywords: Potato, nsLTP, Genome-wide, Tuber development

\section{Background}

Plant non-specific lipid transfer proteins (nsLTPs) were first discovered approximately 35 years ago from spinach leaves [1]. NsLTPs can transfer phospholipids from liposomes to the mitochondria or chloroplast $[1,2]$. In general, plant nsLTPs are small, basic proteins with a size of approximately 6.5 to $10.5 \mathrm{kDa}$, which all have an eight cysteine motif (ECM) [3]. The common form of ECM is

\footnotetext{
*Correspondence: mahaoli@nwsuaf.edu.cn

${ }^{1}$ College of Agronomy, Northwest A\&F University, Yangling 712100, Shaanxi, China

Full list of author information is available at the end of the article
}

C-Xn-C-Xn-CC-Xn-CXC-Xn-C-Xn-C. These cysteine residues can be connected by four disulfide bonds to form a stable hydrophobic cavity structure [4, 5]. According to their molecular masses, the nsLTP proteins were initially divided into two types: nsLTP1 $(9 \mathrm{kDa})$ and nsLTP2 $(7 \mathrm{kDa})$ [1]. Later, depending on the sequence similarity of the ECM, nsLTPs were classified into nine types (Type I, II, III, IV, V, VI, VII, VIII, and IX) [4]. Then, two new types were defined: Type $\mathrm{X}[6]$ and Type XI [7]. The new classification method is not based on molecular size, but rather on the locations of conserved introns, the identity of amino acid sequences, and the

(C) The Author(s). 2019 Open Access This article is distributed under the terms of the Creative Commons Attribution 4.0 International License (http://creativecommons.org/licenses/by/4.0/), which permits unrestricted use, distribution, and 
spacing between Cys residues in the ECM [8]. This classification method is mainly applied to nsLTPs in lower plants [8].

NsLTPs, which account for approximately $4 \%$ of soluble protein, are abundant in higher plants [5]. NsLTPs are involved in many meaningful physiological and biochemical reactions in plant growth, including lipid transport between cell membrane systems, inhibiting the growth of pathogens, signaling and cell wall loosening $[9,10]$. In vitro, plant nsLTPs are able to bind and transfer various types of hydrophobic molecules, including fatty acids, fatty acyl-CoA, phospholipids, glycolipids, and cutin monomers [10-12]. NsLTPs were found to accumulate massively in the apoplastic space and in the vascular system [13], which may be related to adaptation to different environmental stresses [14]. Plant epidermal cells specifically synthesize a waxy cuticle covering all surfaces of the plant to prevent water loss and pathogen intrusion. The cuticle consists of cutin polymers and soluble cuticular lipids [15-17]. To date, an increasing number of nsLTPs' functional studies have been reported in plants. For example, in Arabidopsis, DIR1 encodes a non-specific lipid transfer protein that plays a vital role in plant resistance to the invasion of pathogens $[18,19]$. In some cases, nsLTPs are thought to be associated with pathogenesis-related (PR) proteins. Therefore, it is considered to belong to the PR-14 family [20]. The Arabidopsis nsLTP-encoding gene $A Z I 1$ is involved in signal transduction during bacterial infection [18, 21]. In Arabidopsis, AtLTP2 plays a role in the integrity of the cuticle-cell wall interface and in etiolated hypocotyl permeability [22].

Potato is an important food and economic crop in the world. However, potato yield is susceptible to abiotic and biotic stress [23].In previous studies, overexpression of the AtLTP3 gene enhanced performance of Arabidopsis seedlings under salt, drought, and cold stress [24, 25]. Overexpression of GtLTP1 increases the resistance to Botrytis cinerea, indicating that this gene is a useful molecular tool for enhancing resistance of plants to pathogenic bacteria [26]. Studies have shown that ltpg1 mutants in Arabidopsis are more susceptible to Alternaria brassicicola infection than wild type [27]. In addition, StLTPa7 was shown to be involved in the early stages of resistance to Ralstonia solanacearum via a complex $\mathrm{Ca}^{2+}$-associated expression pathway in potato [28]. In addition, transgenic potato overexpressing StnsLTP1 shows enhanced resistance to various abiotic stresses [29].

Plant non-specific lipid transfer proteins are widely distributed in the plant kingdom and are one of the well-known protein families. In total, 49, 52, and 156 nsLTP members have been identified in Arabidopsis, rice, and wheat, respectively [4]. In the $B$. rapa genome, there are 63 , which can be grouped into nine types based on the diversity of the ECM [7]. In L. japonicus, 25 nsLTPs were identified and divided into seven types (I, II, III, IV, V, VIII, and IX) [30]. A database of 595 nsLTPs from 121 different species has now been established, with classification into five types (I, II, III, IV, V) [31]. Until now, only a small number of potato nsLTPs have been identified [6, 28, $29,32]$. With the completion of the whole-genome sequencing of potato [33], a genome-wide analysis of the StnsLTP genes can now be performed for the first time in our study. In this study, we identified 83 putative StnsLTP genes that can be grouped into eight types in the potato genome. A detailed analysis was carried out, including sequence analysis, phylogenetic relationships, gene structure, chromosomal location, gene duplication, GO annotation, and selective pressure analysis. Based on RNA-seq and the result of qRT-PCR, we performed the expression profiling of StnsLTPs in different tissues. The results obtained from this study laid the foundation for the further study of the function of StnsLTPs in potato, especially some genes that may have important functions in antibiotic processes and abiotic stress.

\section{Materials and methods}

\section{Identification of $n s L T P$ family members in potato}

To identify candidate members of the StnsLTP family, several searches were conducted. First, the potato proteome and genome sequence were downloaded from the PGSC database (http://solanaceae.plantbiology.msu.edu/ pgsc_download.shtml) [33]. Second, the published Arabidopsis and rice nsLTP sequences were retrieved and used as queries to identify the potato nsLTPs via the BLASTp and tBLASTn searches with a cutoff E-values of $1 \mathrm{e}^{-10}$ and $\mathrm{e}^{-3}$, respectively. In addition, we searched the PGSC database by name search using the name "nsLTPs" and "non-specific lipid transfer protein" as queries. To avoid missing potential StnsLTP members, we downloaded the HMM profile of plant lipid transfer proteins (PF00234) from the Pfam (http://pfam.xfam. org/) and used it as a query to for hmmsearch against the potato protein database by the HMMER 3.0 package [34] with the default parameters. After integrating the results of the above searches and eliminating redundancy, we also removed nsLTPs that did not contain an ECM domain. Additionally, within the candidate potato nsLTP proteins, a BLASTp search was performed on the protein sequences of $2 \mathrm{~S}$-albumin (At2S1 to At2S4) [35] and alpha-amylase inhibitor (RATI) [36] to exclude possible cereal storage proteins.

Finally, the potential nsLTP protein sequences were analyzed using the NCBI-CDD (https://www.ncbi.nlm. nih.gov/Structure/bwrpsb/bwrpsb.cgi) and Pfam websites. The sequences with incomplete domains and lack of nsLTP annotation were removed. The identified 
StnsLTPs were named in term of the method proposed by Boutrot et al. [4].

\begin{abstract}
Multiple sequence alignment and phylogenetic analysis Multiple sequence alignment of the conserved ECM domains of the StnsLTPs was performed using ClustalX version 2.1 software with the default settings. Then, a phylogenetic tree was constructed by MEGA7 (http:// www.megasoftware.net/) using the Neighbor-Joining (NJ) method with parameters as follows: Each node was calculated using 1000 repeated bootstrap tests [37], and the model/method was p-distance, and the gaps/ missing data treatment was pairwise deletion.
\end{abstract}

\section{Identification of conserved motifs and gene structures in potato nsLTPs}

To identify conserved motifs in the StnsLTPs, the MEME website (http://meme-suite.org/tools/meme) was used for identification with the default parameters except for the maximum number of motifs, which was set to 10 .

The exon-intron structures of the StnsLTP genes were revealed using the GSDS2.0 website (http://gsds.cbi.pku. edu.cn/index.php). The genomic sequence and CDS sequence of the same StnsLTP gene were simultaneously submitted to the GSDS website to reveal its gene structure.

\section{Chromosomal locations and gene duplications of potato nsLTPs}

Information about all the identified StnsLTPs was retrieved from the PGSC website. MapChart2.3 software (https://www.wur.nl/en/show/Mapchart.htm) was used to map the chromosomal positions and relative distances of the StnsLTP genes. In general, genes separated by no more than five other genes within $100 \mathrm{~kb}$ were considered as tandem duplications [38]. The segmentally duplicated StnsLTP genes were identified on the PGDD website (http://chibba.agtec.uga.edu/duplication/).

\section{Selective pressure analysis}

The coding sequences for the duplicated genes were aligned by MEGA6 using the Muscle (codon) method. KaKs_Calculator2.0 Software [39] was used to calculate the $\mathrm{Ka}$ (the number of nonsynonymous substitutions per nonsynonymous site) and Ks (the number of synonymous substitutions per synonymous site) of duplicated genes by the MYN method. The divergence time " $\mathrm{t}=\mathrm{Ks} / 2 \mathrm{r}$ " was calculated with the neutral exchange rate $r=2.6 \times 10^{-9}$.

\section{Identification of cis-acting elements of potato nsLTPs}

To investigate the cis-acting elements, the upstream regions of all the StnsLTP genes $(1500 \mathrm{bp})$ were extracted from the Phytozome website (https://phytozome.jgi.doe. gov/pz/portal.html). Then, all of the sequences were submitted to the PlantCARE website (http://bioinformatics.psb.ugent.be/webtools/plantcare/html/) [40] to identify possible cis-acting elements.

\section{GO annotation and RNA-seq data analysis}

To better comprehend the function of the StnsLTP genes, Blast2GO software (Version5.0) [41] was used to perform gene ontology (GO) analysis under the default parameters according to the following steps: (i) The full length amino acid sequences of the StnsLTPs were loaded into the Blast2GO software; [42] (ii) The BLASTp program built into the Blast2GO software was used to search the NCBI non-redundant protein database with the cutoff e-value $\left(1 \mathrm{e}^{-10}\right)$; (iii) StnsLTP genes were mapped based on the BLASTp results; (iv) StnsLTPs were annotated. The annotation contained three categories: molecular function, cellular component, and biological process.

Processed RNA-seq data were downloaded from the PGSC database. The RNA-seq data of different developmental stages and different tissues of potato were selected to study the spatiotemporal specificity of StnsLTP gene expression. The expression patterns of StnsLTPs were analyzed in two varieties, DM (doubled monoploid) and RH (heterozygous diploid) [33]. The transcript abundance of the StnsLTP genes was represented by the FPKM (fragments per kilobase of exon per million fragments mapped) values. The heatmap of the StnsLTP genes was generated by the pheatmap package (https://CRAN.R-project.org/package=pheatmap). Genes with an FPKM value of zero in all tissues were excluded from the heat map.

\section{Plant materials}

Potatoes (S. tuberosum cv. Atlantic), which were maintained in Dr. Chen's laboratory at Northwest A \& F University, were planted in a greenhouse at Northwest A \& F University. During the growth of the potato, conventional cultivation techniques were used. Roots, leaves, stems, flowers, young tubers, and mature tubers were collected from potato plants at the young flowering stage to analyze the tissue-specific expression patterns of the StnsLTP genes. The different tuber developmental stages, including stolon (S1), tuber of the initial stage (S3), and mature tuber (S8), were defined as previously described [43]. In addition, there were at least three biological replicates and two technical replicates per tissue or organ. The collected samples were frozen without delay in liquid nitrogen and stockpiled at $-80^{\circ} \mathrm{C}$ for later use.

\section{Quantitative RT-PCR analysis}

Total RNA was extracted from the previously collected samples using the RNAsimple Total RNA Kit (BioTeke, 
Beijing, China). According to the manual of the RNA purification kit, the genome DNA contamination was digest in the RNA binding membrane with RNase free DNase I. The cDNA, which was synthesized by the PrimeScript reagent kit (Takara, Japan) in accordance with the manufacturer's instructions, was diluted 5-fold for future analysis. Gene-specific primers for qRT-PCR were designed by Primer Premier 6 software (http://www.premierbiosoft.com/primerdesign/), which are listed in Additional file 1: Table S1. The qRT-PCR was performed on the Bio-Rad real-time system (CFX96, USA) using the KAPA SYBR FAST qPCR Kit Master Mix (2x) Universal (KAPA BIOSYSTEMS, Boston, United States). Three biological replicates and two technical replicates were analyzed. The qRT-PCR amplification system and amplification procedure were as previously described [44]. The internal reference gene was ef1 $\alpha$. The StnsLTP expression levels from different RNA samples were normalized as described previously [45]. The relative expression levels of the StnsLTP genes were calculated using a standard curve and normalized as described previously [46]. The results are shown as the mean \pm standard deviation (SD).

\section{Results}

\section{Genome-wide identification of StnsLTP genes}

To identify all StnsLTP genes in potato, four bioinformatics methods were used in this study. In total, 77, 33, 54, and 128 StnsLTP genes were identified by keyword search, local BlastP search, local tBlastn search, and HMM search, respectively. After integrating the four parts of the results, sequences that contain incomplete ECM domains were manually deleted. Subsequently, 21 proteins translated by non-representative transcripts were removed. Next, a $2 \mathrm{~S}$ albumin storage protein was also discarded. In addition, seven proteins that were annotated in the NCBI-CDD as incomplete proteins were also discarded. As a result, 83 sequences were identified as StnsLTP genes.

In previous studies, 28 StnsLTP genes were identified $[6,28,29]$. Then, 83 sequences were established based on the classification method (Table 1). However, several sequences could not be classified by the previous method. Hence, according to the similarity of the ECM domain sequences in amino acid sequences, those sequences were classified into two new types, XII and XIII. The genes were named according to their positions on the chromosome (Additional file 2: Table S2). The majority of the StnsLTPs had 0-2 introns, and only two genes (StnsLTPIV.8, StnsLTPIV.10) had 3 introns. The CDS sizes of the StnsLTPs ranged from 276 (StnsLTPII.2) to $666 \mathrm{bp}$ (StnsLTPIV.8). Eight StnsLTPs (StnsLTPI.6, StnsLTPI.19, StnsLTPI.24, StnsLTPI.28, StnsLTPVII.1, StnsLTPVIII.7, StnsLTPXII.1, StnsLTPXIII.6) lacked an $\mathrm{N}$-terminal signal peptide. The 83 identified StnsLTP proteins ranged in length from 92 (StnsLTPII.2) to 221 (StnsLTPIV.8) amino acids (aa) and had molecular weights $(\mathrm{Mw})$ ranging from $9.77 \mathrm{kDa}$ (StnsLTPII.1) to $22.88 \mathrm{kDa}$ (StnsLTPIV.8). The theoretical isoelectric points (pI) were predicted from 4.16 (StnsLTPXII.2, StnsLTPXII.4) to 9.76 (StnsLTPIV.3).

\section{Sequence analysis and classification of putative StnsLTPS}

Because the potato is a flowering plant [8], we used the sequence similarity method to classify StnsLTPs. Based on the classification scheme of Boutrot et al. (2008) and Liu et al. (2010), we added two new types of nsLTP (types XII and XIII) based on the sequence similarity of the ECM $[4,6]$. The 83 StnsLTPs were divided into 8 types (I, II, IV, V, VII, VIII, XII, and XIII); none were classified as type III, VI, X, or XI (Table 1 and Additional file 2: Table S2). Interestingly, type II potato nsLTPs were single-exon genes. This result was consistent with other studies in B. rapa, Gossypium spp. and Solanum lycopersicum $[12,32]$. As shown in Table 1, the difference between the new type XII and XIII was mainly the number of amino acids between the fourth cysteine residue and the fifth cysteine residue. The eight Cys residues in all 83 StnsLTPs were found to be highly conserved in structure (Fig. 1 and Additional file 5: Figure S1). Of the 83 StnsLTP genes we identified, the members of type I accounted for the largest proportion (43.37\%).

Table 1 Some characteristics for the different types of non-specific lipid transfer proteins found in potato

\begin{tabular}{|c|c|c|c|c|c|c|c|c|c|c|c|c|}
\hline Type & Number of members & & & & & & Spacing $\mathrm{p}$ & & & & & \\
\hline I & 36 & C & $X_{9-10}$ & C & $X_{12-17}$ & CC & $X_{19}$ & $C X C$ & $X_{20-24}$ & C & $x_{3,13,14}$ & C \\
\hline$\|$ & 6 & C & $x_{7}$ & C & $x_{13}$ & CC & $x_{8}$ & $C X C$ & $x_{23,21}$ & C & $x_{6}$ & C \\
\hline IV & 10 & C & $X_{9,10,18}$ & C & $X_{14-17}$ & CC & $x_{9,12,20}$ & $C X C$ & $x_{24}$ & C & $X_{6-10,13}$ & C \\
\hline V & 2 & C & $X_{14}$ & C & $X_{14}$ & CC & $X_{11}$ & $C X C$ & $x_{24}$ & C & $X_{10}$ & C \\
\hline VII & 1 & C & $x_{6}$ & C & $X_{14}$ & CC & $x_{12}$ & $C X C$ & $X_{27}$ & C & $x_{8}$ & C \\
\hline VIII & 12 & C & $x_{6,9}$ & C & $X_{12-14,16}$ & CC & $x_{12}$ & $C \times C$ & $x_{25}$ & C & $x_{8,9}$ & C \\
\hline XII & 7 & C & $x_{9,10}$ & C & $X_{13,14}$ & CC & $x_{20,21}$ & $C \times C$ & $x_{21}$ & C & $X_{9,12,13}$ & C \\
\hline XIII & 9 & C & $x_{8,9}$ & C & $X_{13-16}$ & CC & $X_{8,9,12,21}$ & $C X C$ & $X_{19,21-23,26,30}$ & C & $X_{6-11,13}$ & C \\
\hline
\end{tabular}

The character " $X$ " represents any kind of amino acid, and the Arabic numbers following " $X$ " represent the number of amino acid residues 


\section{Chromosomal locations and gene duplications of StnsLTP genes}

To demonstrate the exact locations and distributions of the StnsLTP genes on different chromosomes, we constructed a detailed chromosome map. The positions of the StnsLTP genes were downloaded from the PGSC database. Mapchart software version 2.3 was used to generate the chromosome map. Seven genes, including StnsLTPI.1, StnsLTPI.2, StnsLTPI.3, StnsLTPI.4, StnsLTPI.5, StnsLTPI.6, and StnsLTPIV.3, could not be mapped to any of the potato chromosomes. The rest of the StnsLTPs were randomly distributed on ten chromosomes, with none on chromosomes 04 and 12. The largest number of StnsLTP genes were found on chromosome 06 (14 genes). In addition, 13 StnsLTP genes were located on chromosome 01; 11 members on chromosomes 03 and 08; two members on chromosomes $02,05,07$, and 11; five on chromosome $09 ; 12$ on chromosome 10 (Fig. 2).

In the process of evolution, both tandem duplication and segmental duplication are a pivotal part in the generation of gene families [47]. Therefore, StnsLTP gene duplication events were analyzed. According to the general criteria (two genes are separated by fewer than five other genes within a $100 \mathrm{~kb}$ chromosome fragment [38]), 16 gene clusters were regarded as tandem-duplicated genes, accounting for $53.01 \%$ (44 out of 83). According to the data downloaded from the PGDD website, three pairs of StnsLTP genes were identified as segmentally duplicated genes (Fig. 2).

In addition, the $\mathrm{Ka} / \mathrm{Ks}$ value for each pair of duplicated genes was calculated to estimate the rate of molecular evolution (Additional file 3: Table S3). The value of $\mathrm{Ka} /$ $\mathrm{Ks}$ is conventionally used as a sign of selection pressure [48]. $\mathrm{Ka} / \mathrm{Ks}>1$ indicates a positive selection effect, while $\mathrm{Ka} / \mathrm{Ks}<1$ indicates that a purifying selection exists between the duplicated genes; $\mathrm{Ka} / \mathrm{Ks}=1$ suggests that a neutral selection exists between the duplicated genes [49]. Our results showed that $91.49 \%$ of the duplicated StnsLTPS underwent purifying selection pressure during the duplication events (Additional file 3: Table S3), which showed that the function of duplicated StnsLTPs might not have changed much in the subsequent evolutionary process. In addition, the divergence time between duplicated gene pairs was also calculated using the previously described formula (Additional file 3: Table S3). The Ks values of the vast majority of StnsLTPs were greater than 0.52 and the corresponding divergence time might be greater than 100 million years ago (MYA). Interestingly, the $\mathrm{Ks}$ value of one tandem duplicated gene pair (StnsLTPI.24/StnsLTPI.21) was 4.92384, and the corresponding duplication age might be 946.89 million years ago (MYA) (Additional file 3: Table S3).

\section{Phylogenetic analysis of potato, rice and Arabidopsis nsLTPs}

To better understand the evolutionary relationship among nsLTPs, a phylogenetic analysis of nsLTPs identified in potato, Arabidopsis, and rice was performed using ClustalX2.1 and MEGA7 software. In total, 177 nsLTPs from the three species were analyzed. We performed multiple sequence alignments with previously identified ECM conserved domain sequences of potato, rice, and Arabidopsis. A phylogenetic tree was constructed by the NJ method as described above (Fig. 3). It was noteworthy that nsLTPs were more closely related to members of the same type than other types of nsLTPs from the same species, suggesting a relatively high degree of homology within the same type of nsLTPs from different species. Among the eight types of StnsLTPs, only the distributions of only type XIII and IV were relatively random. Therefore, the two new types of StnsLTPs may have evolved from other types of StnsLTPs.

\section{Conserved protein motifs and gene structure of potato nsLTPs}

The major feature of plant nsLTPs is the highly conserved ECM. Using the ECM sequences of the nsLTPs identified

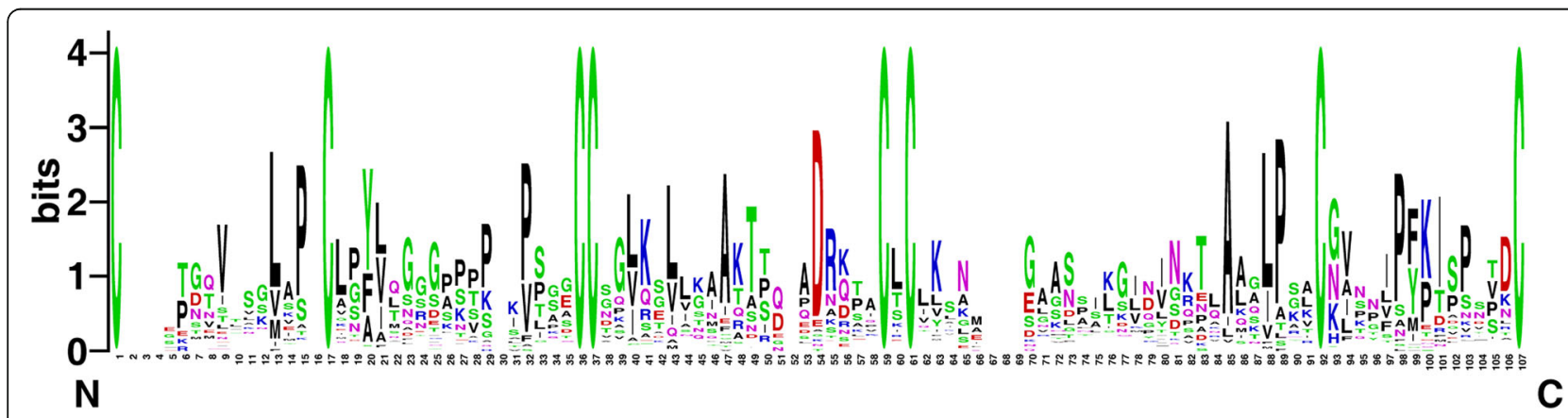

Fig. 1 StnsLTPs were analyzed in a conservative domain through the WebLogo website. The height of the amino acid residue at each position represents the degree of conservation of this amino acid residue. The number on the $x$-axis indicates its sequence position in the corresponding conserved domain. The $y$-axis represents information measured in bits 


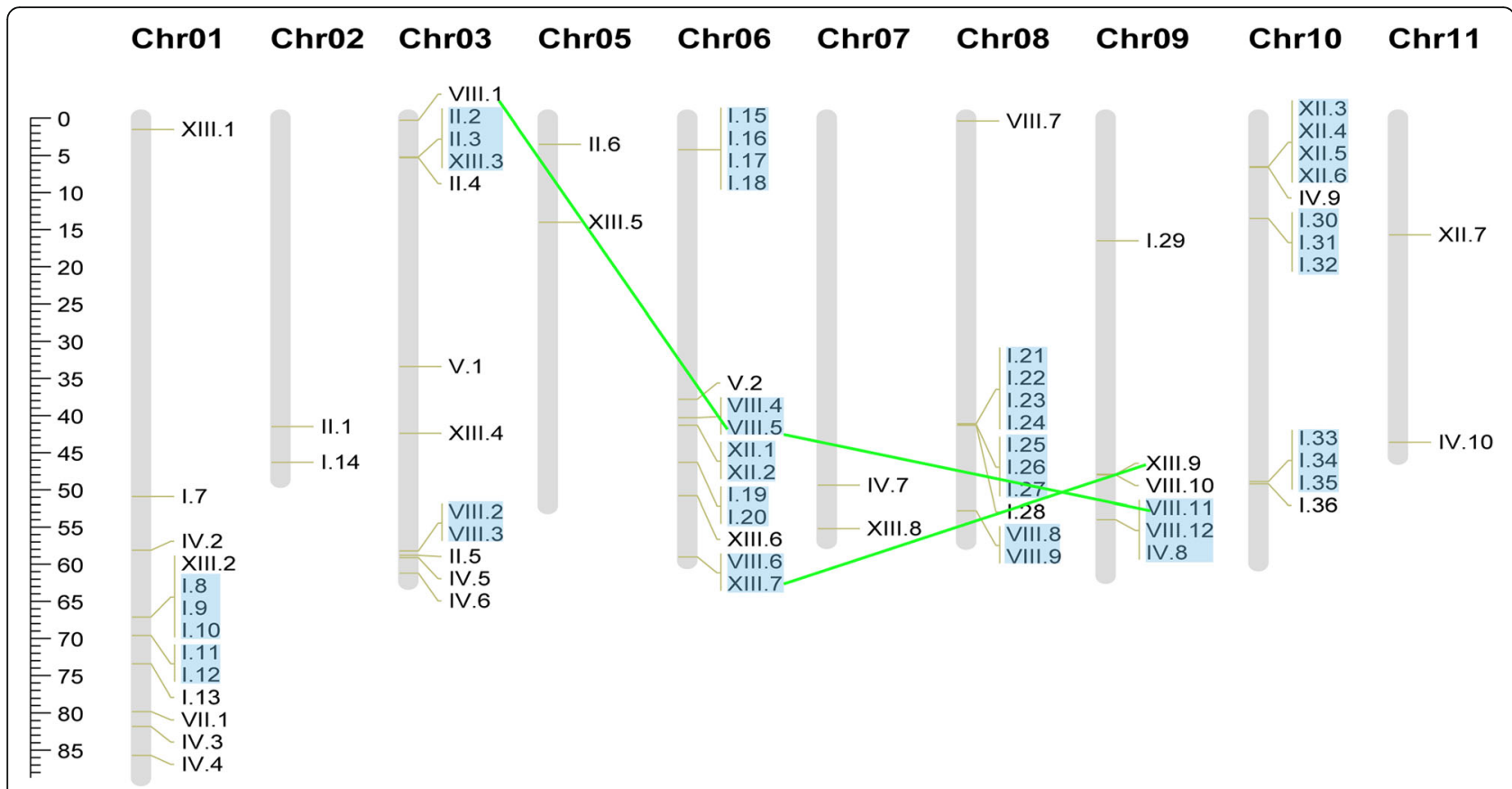

Fig. 2 Chromosomal localization and gene duplication of StnsLTPS. The tandem duplicated genes are marked with blue rectangles, and the segmentally duplicated genes are linked by green lines. The positions of the StnsLTP genes on the chromosome can be inferred from the ruler on the left, and the units of the ruler is megabases $(\mathrm{Mb})$

in potato, sequence logos were visualized by the WebLogo website (http://weblogo.berkeley.edu/logo.cgi) (Fig. 1). Then, to better understand motif conservation in StnsLTPs, ten motifs were identified by the MEME website (Fig. 4c). In addition, the results of the multiple sequence alignment showed a change in the number of amino acid residues between the eight conserved cysteines, which contributed to the classification of different types of StnsLTPs (Additional file 5: Figure S1).

Gene organization takes part in the evolution of multigene families [50]. Therefore, the intron-exon structure of the 83 StnsLTP genes were analyzed. As shown in Fig. $4 \mathrm{~b}$, type I, which included more than half $(52.79 \%)$ of the StnsLTP genes, didn't have intron, and the remaining member of StnsLTPs had one intron. Interestingly, members of type II didn't contain intron, members of type $\mathrm{V}$ and VII had only one intron, and type IV members had a wide variety of intron structures. There was one intron in StnsLTPIV.8, two introns in StnsLTPIV.4 and StnsLTPIV.6, and three introns in StnsLTPIV.7, StnsLTPIV.9, and StnsLTPIV.11. The remaining five members didn't have intron in their gene structure. In type XII, only StnsLTPXII.1 had one intron, and the remaining members didn't have intron. In type XIII, there was one intron in StnsLTPXIII.5 and StnsLTPXIII.6, two introns in StnsLTPXIII.1, StnsLTPXIII.2 and StnsLTPXIII.7, and the remaining genes didn't have intron. In summary, members of the StnsLTP gene family contained small numbers of introns (maximum of three) or didn't have intron.

\section{Gene ontology analysis of potato nsLTPs}

To better understand the molecular functions, biological processes and cellular components of StnsLTPs, gene ontology (GO) analysis was performed. As shown in Fig. 5 and Additional file 4: Table S4, only 48 StnsLTP proteins were annotated in terms of molecular function. In total, 47 StnsLTPs were annotated as lipid binding. Surprisingly, StnsLTPIV.9 was annotated as kinase activity. Only 62 StnsLTPs were annotated in the category of biological process. The annotated StnsLTPs were involved in a variety of biological processes. Most StnsLTPs (96.77\%) took part in lipid transport. In addition, StnsLTPI.29 could participate in multiple biological processes, such as defense response and response to biological stimuli. It is noteworthy that the involvement of StnsLTPIV.9 in the phosphorylation process was related to its molecular function. Based on the biological process analysis, the main functions of StnsLTPs were to bind lipids and participate in some other biological processes such as abiotic and biotic stress responses and signal transduction. In addition, 55 StnsLTPs were annotated in a cellular component. The results showed that StnsLTPs were located on the cell membrane, which was related to their function of transporting lipids. 


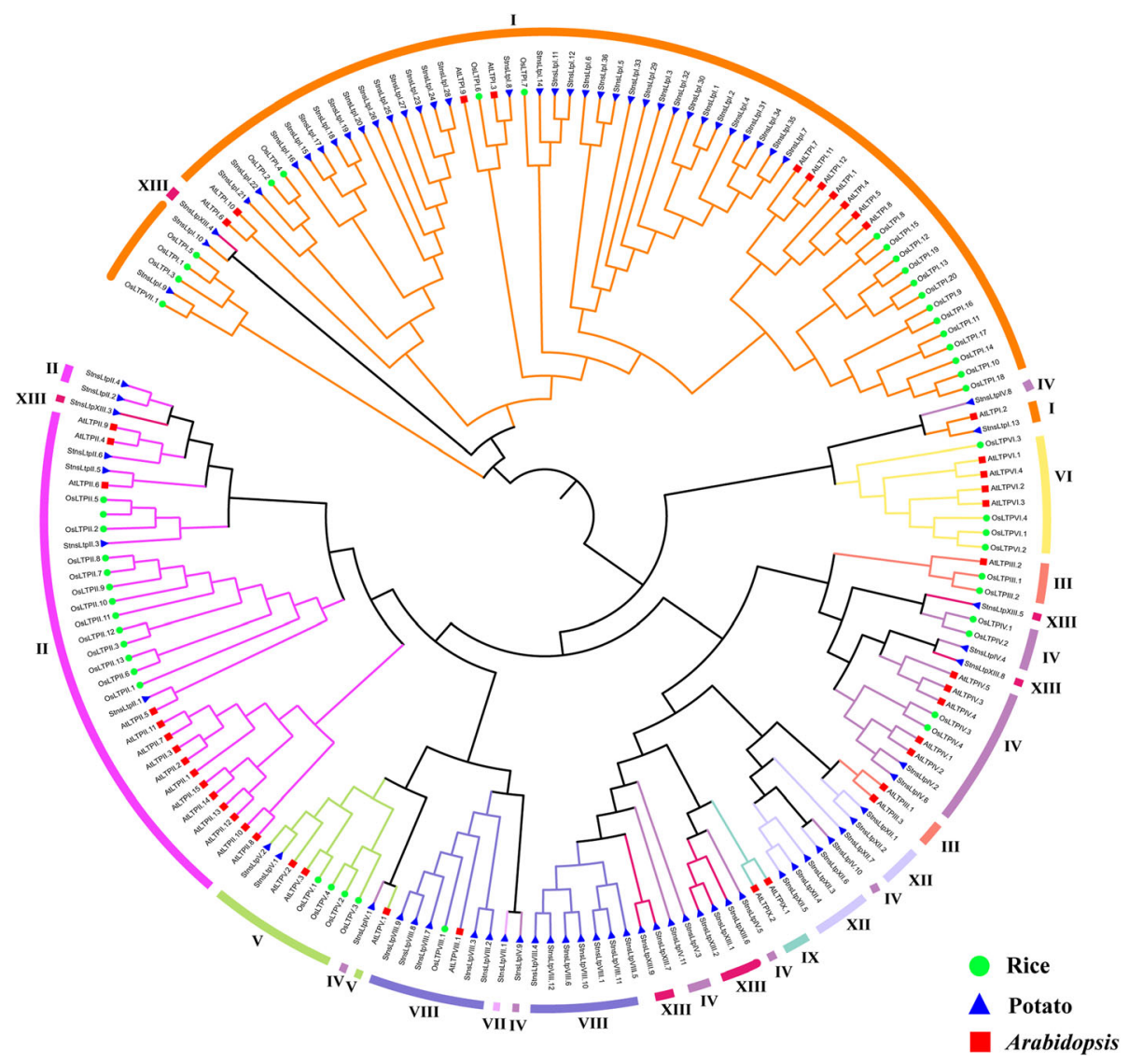

Fig. 3 A phylogenetic tree of Arabidopsis, rice, and potato nsLTP proteins. A phylogenetic tree was constructed using the NJ method using MEGA7 software, and 1000 bootstrap tests were performed. Different types are marked with different colors

Key cis-acting elements within the promoter regions of StnsLTPs

Cis-acting elements that located in the promoter are the binding sites for transcription factors, and thus, they are also essential for regulating the initiation of gene transcription [40]. To further explore the regulatory mechanisms of StnsLTPs in development and abiotic and biotic stress response, a 1500 bp upstream sequence from each transcription start site was submitted to the PlantCARE website [40] for identification of cis-acting elements. Because potato is an endosperm-free species, we did not show cis-acting elements associated with endosperm development in the results. As shown in Additional file 6: Figure S2, 18 types of cis-acting elements were identified. The StnsLTPs contained a large number of stress-responsive cis-acting elements. All cis-acting elements could be divided into three types according to their different functions. Type A cis-acting elements were all related to plant hormones, including P-box, CGTCA-motif, TGACG-motif, ERE, TGA-element, and TATC-box. Type $\mathrm{B}$ cis-acting elements were related to plant response to biotic and abiotic stress, including Box-W1, WUN-motif, TC-rich repeats, LTR, MBS, HSE, and TCA-element.
Type C cis-acting elements were related to regulating plant growth and development, including ARE, CCGT CC-box, CAT-box, circadian, and 5UTR Py-rich stretch.

\section{Expression patterns of StnsLTP genes}

To better elucidate the expression patterns of StnsLTPs, we analyzed the public RNA-seq (RNA sequencing) data available on the PGSC website. A heatmap was generated except for StnsLTPI.20, for which the FPKM value could not be found. As shown in Fig. 6a and b, the expression of the StnsLTPs showed temporal and spatial specificity and with a slight difference between varieties, which may be due to different genetic backgrounds. Interestingly, most of the StnsLTP genes were expressed in flower organs in both DM and RH. Most of the StnsLTP genes were highly expressed only in a specific organ or at a specific developmental stage. For example, the StnsLTPV.1, StnsLTPXIII.9, StnsLTPV.2, and StnsLTPVIII.12 genes have high expression in the root but low expression in other organs. Similar to previous studies, the StnsLTPVIII.1, StnsLTPXIII.4, and StnsLTPVIII.8 genes were found in young potato tubers and were specifically expressed at low levels in mature potato tubers. 


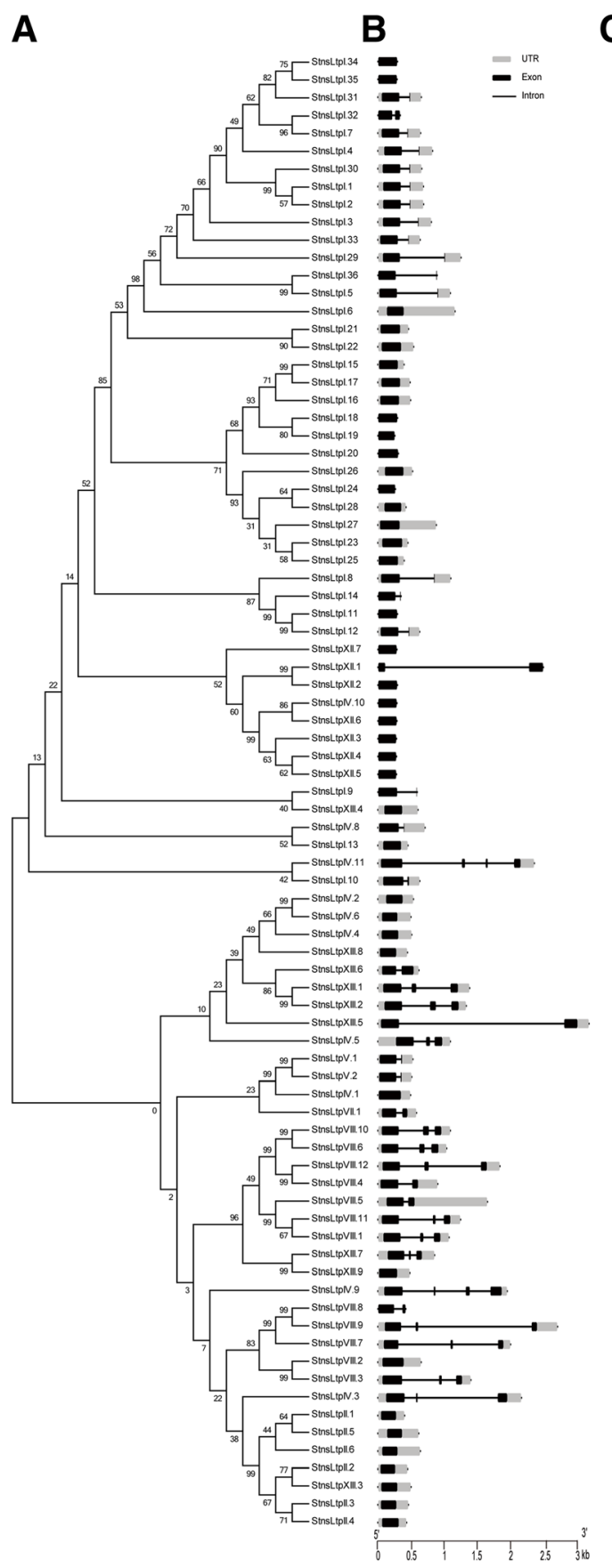

C

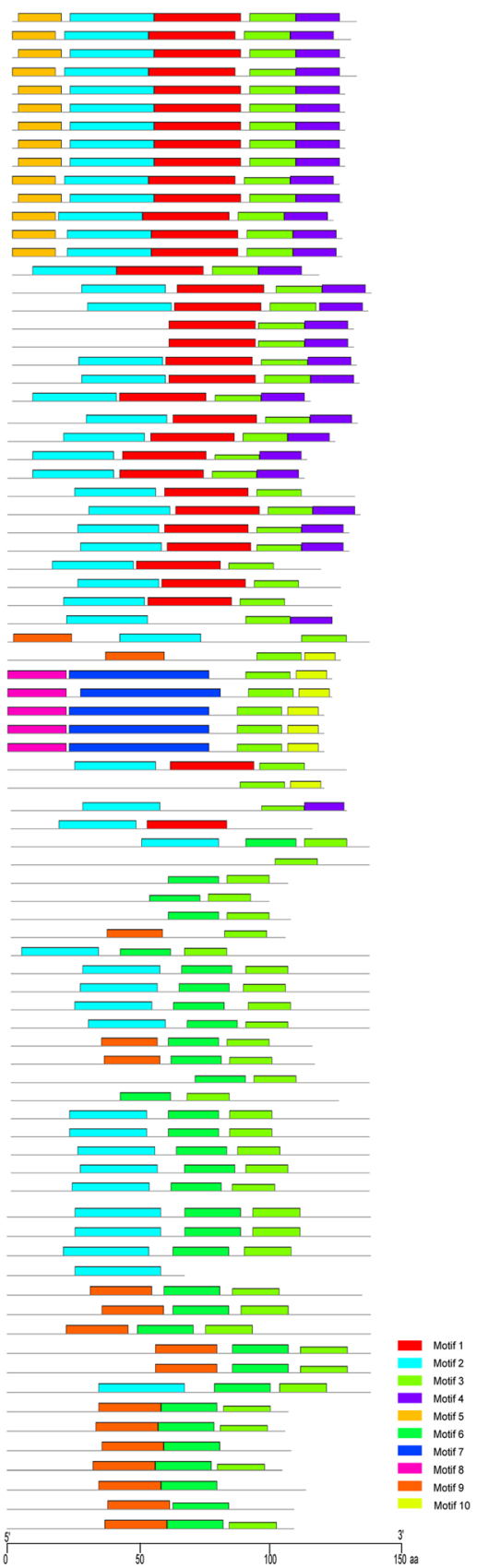

Fig. 4 Phylogenetic relationships and gene structure of the StnsLTP genes and conserved motifs of the StnsLTP proteins. A, Phylogenetic tree of 83 StnsLTP proteins. A rootless Neighbor-Joining phylogenetic tree was constructed using MEGA7 software with the full-length amino acid sequences of 83 StnsLTP proteins, and the bootstrap value was set to 1000. B, A conserved motif distribution map in the StnsLTP gene. The 10 predicted motifs are represented by different colored boxes. C, Exon/intron maps of StnsLTP genes. Black boxes represent exons, and black lines represent introns. The UTRs region of the StnsLTP genes are represented by a gray box. The lengths of the introns and exons can be inferred by the ruler at the bottom

\section{Expression analysis of StnsLTPs by quantitative RT-PCR}

To better understand the biological roles of the StnsLTP genes, 22 StnsLTP genes were selected based on RNA-seq data and gene-specific primers were designed. Six tissues, including young leaf (Lf), root (R), stolon (Sn), young tuber (YT), mature tuber (MT), and flower (F) of potato cultivar Atlantic, were analyzed by quantitative RT-PCR. As shown in Fig. 7, on the basis of their expression patterns, these 22 StnsLTP genes were divided into five groups (A-E). 


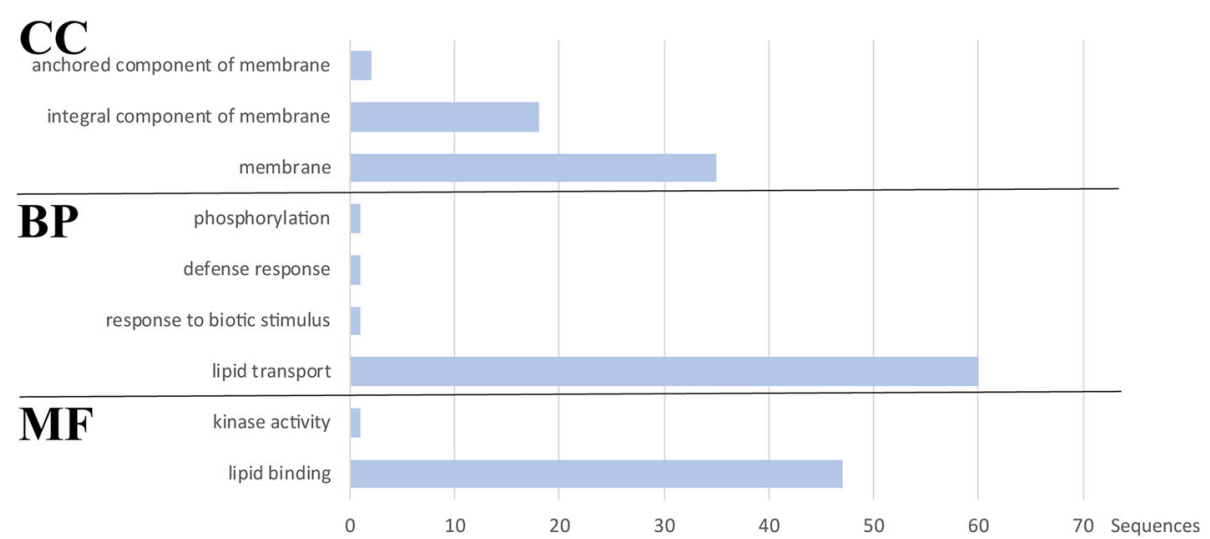

Fig. 5 Gene Ontology (GO) annotation of the StnsLTP protein. The result of the annotation includes three parts: MF, molecular function. BP, biological process. CC, cell composition

a

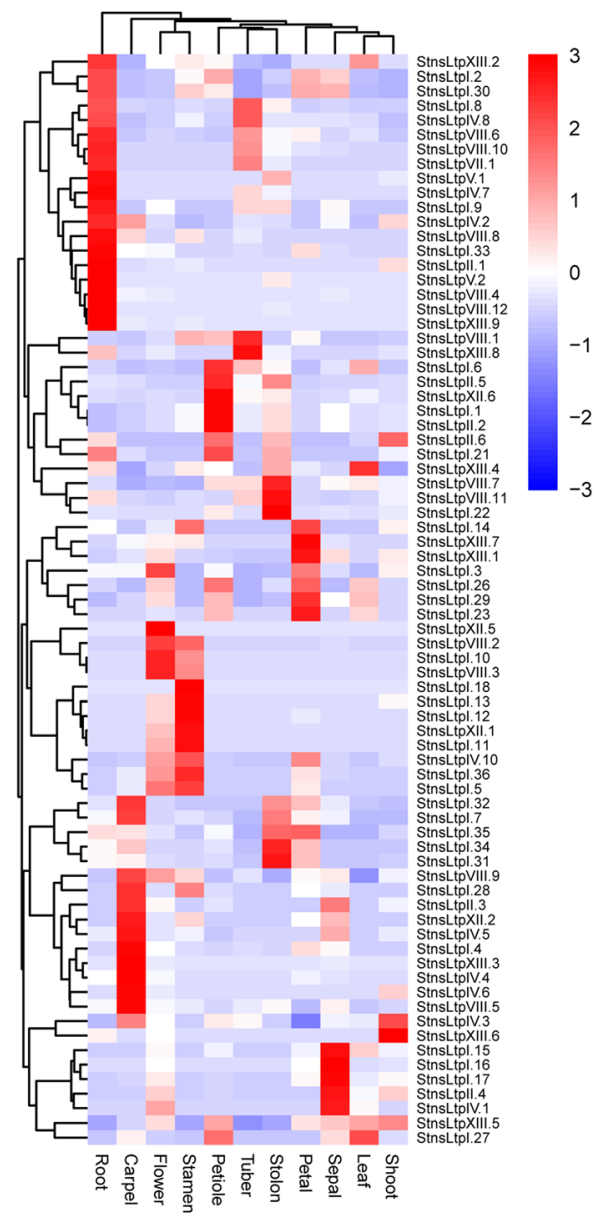

b

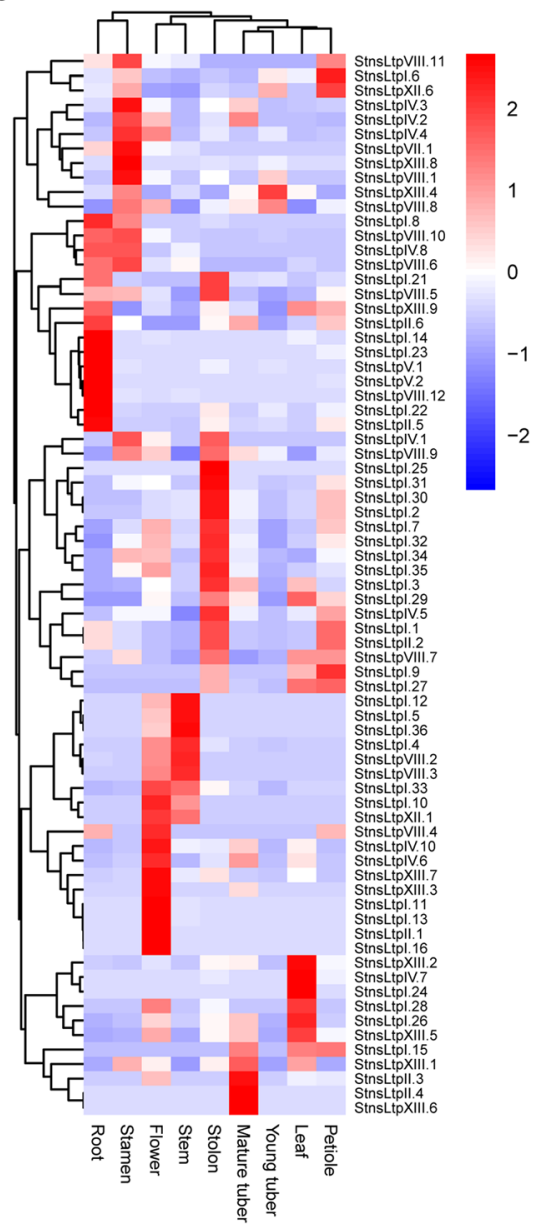

Fig. 6 Expression profiles of StnsLTP genes in double monoploid (a) and heterozygous diploid (b). The FPKM value of the StnsLTP gene was generated from the heatmap of the pheatmap package in the $\mathrm{R}$ software 


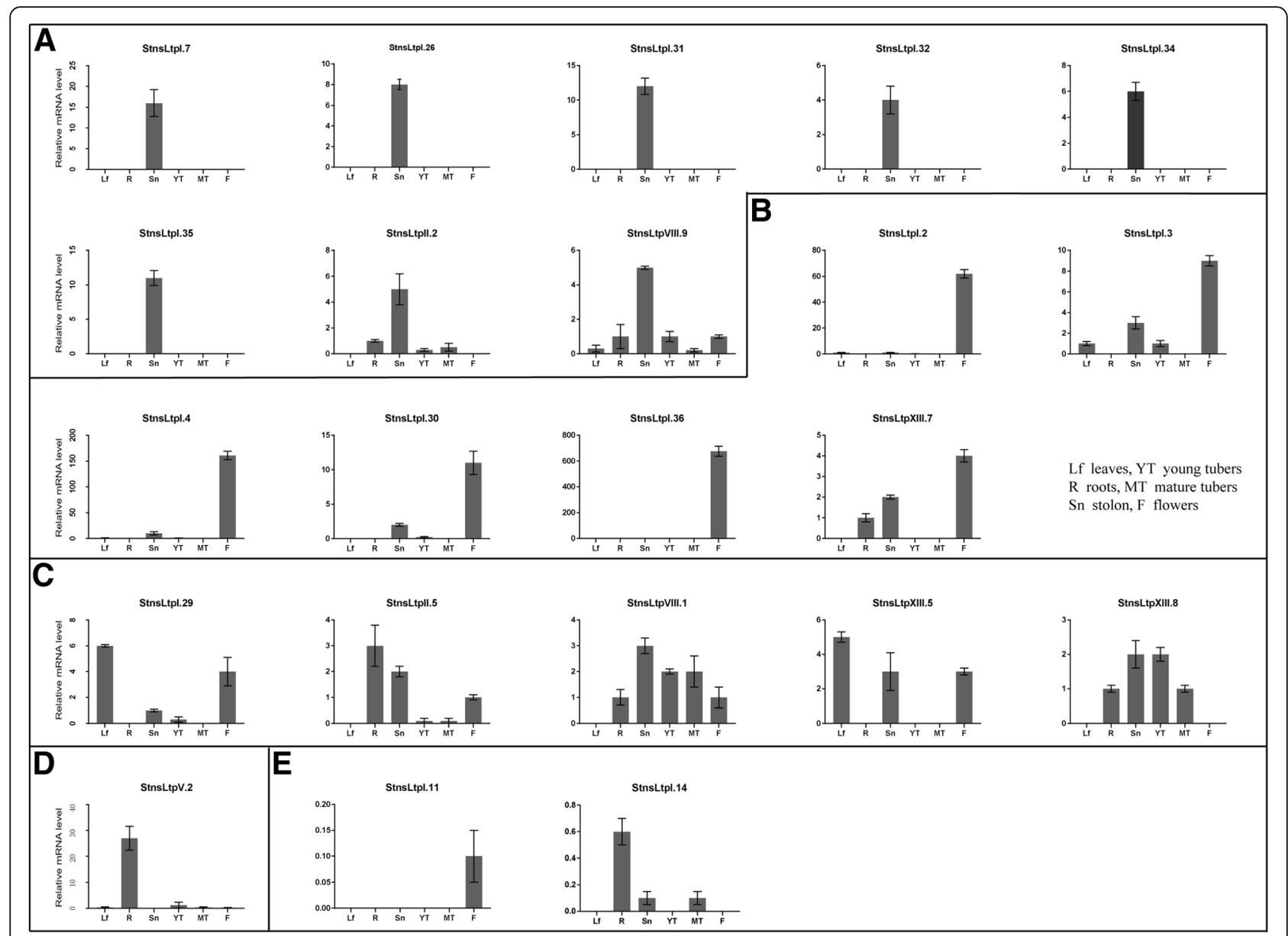

Fig. 7 Expression profiles of StnsLTP genes in six tissues. Quantitative RT-PCR was used to study the expression levels of 22 selected StnsLTP genes. The results were expressed as the mean \pm standard deviation. The internal reference gene used in qRT-PCR was efia

Genes of group A were specifically expressed in the stolon. Among them, StnsLTPI.7, StnsLTPI.26, StnsLTPI.31, StnsLTPI.32, StnsLTPI.24. and StnsLTPI.35 were mainly expressed in the stolon, while the other two genes (StnsLTPII.2 and StnsLTPVIII.9) were also expressed in other organs. The genes of group B were specifically expressed in the flower. Compared with the other analyzed genes, the $\mathrm{C}$ group of genes showed diverse expression patterns with expression in multiple tissues. For example, StnsLTPI.29 had the highest expression level in the leaf, but it also showed some expression in the flower and other tissues. StnsLTPV.2, which was the only gene in group $\mathrm{D}$, was mainly expressed in the root. Both genes of the $\mathrm{E}$ group were barely expressed in all the sampled organs. The expression levels of StnsLTPI.11 and StnsLTPI.14 in the six organs were very low, suggesting that they might have a special spatiotemporal expression pattern, but they were not detected in the qRT-PCR data.

\section{Discussion}

Currently, potatoes are an important part of the world's food supply. However, during growth and development, potatoes are subjected to various stresses [51, 52]. Plant nsLTPs, which are abundant in higher plants, account for approximately $4 \%$ of soluble protein and play a unique role in plant life activities [22, 29, 53, 54]. This gene family has been identified in Arabidopsis, rice, wheat, maize, $B$. rapa and other plants $[4,7,10,32,42]$. However, little is known about the nsLTP gene family in potato, because the potato genome-wide sequence has only recently been published [33].

In this study, we identified 83 potential potato $n s L T P$ genes in silico, which were classified into eight types (I, II, IV, V, VII, VIII, XII, and XIII), of which the XII and XIII types are new types identified herein; no Type III, VI, IX and XI nsLTPs were found in potato. A previous study of six Solanaceae species showed that four types of $n s L T P$ (III/V/VI/VIII) were not identified [6]. Our results are similar to the previous study. However, there 
are 12 nsLTPs of type VIII in this study. Why do Type III/V/VI nsLTPs have so few members, while the number of type VIII nsLTPs suddenly increased in the potato genome? This is still a question that needs further study. It is worth noting that we identified a gene belonging to type VII, which contains 27 amino acid residues between Cys6 and Cys7, although it is speculated that type VII nsLTPs occur only in monocots $[4,6]$. Therefore, we believe that the first reason is the lack of precise mapping of genome sequencing, and the second reason is due to the complexity of the potato genome.

Although many potential nsLTPs have been identified based on the whole-genome sequences in the plant kingdom, only a small number of nsLTP functions have thus far been elucidated. As the research progresses, increasing evidence has shown that nsLTPs are likely involved in many biological processes, such as signal transduction, reproduction, and response to biotic and abiotic stresses [3]. Unfortunately, the potato is often subjected to abiotic stress, which in turn leads to poor plant growth and ultimately yield loss. For example, when the potato is exposed to water stress during the development period, its yield will decrease as the stress level deepens [55].

As an evolutionary relic, intron-exon arrangements bear the marks of gene family evolution [56, 57]. This is consistent with the results previously reported by researchers that plants tend to retain genes that do not contain introns or contain short introns during evolution [58]. The nsLTP family formed during the early differentiation of terrestrial plants and evolved new subclasses during terrestrial plant evolution $[5,42]$. Unlike animals, genes with few or no introns in plants are thought to be expressed at lower levels [59]. However, a compact genetic structure may help genes to respond to endogenous and/or exogenous stimuli by allowing rapid expression [60].

The character of genetic duplication in the origins of evolutionary novelty, complexity and the expansion of gene families has long been recognized [56, 57, 61]. The major duplication patterns reported are tandem duplication and segmental duplication [62]. In our findings, both tandem and segmental duplication events played an important role in potato nsLTP amplification. StnsLTPs are located on ten potato chromosomes. The number of nsLTPs identified in potato exceeds those previously identified in Arabidopsis, rice, wheat, and maize. Although the genome size of potato $(844 \mathrm{Mb})$ [33]is close to seven times as that of Arabidopsis(125 $\mathrm{Mb})$ [63], the number of nsLTP genes in potato (83 genes) is 1.69 times the number of nsLTP genes in Arabidopsis ( 49 genes). The key reason for this visible fact is that the evolution of this gene family may be complex and affected by gene duplication and gene loss. The
nSLTP gene family may have lost genes during the whole-genome duplication of the potato. A grand total of 19 duplicated StnsLTPs gene pairs were found, including 16 tandem duplicated gene clusters and three pairs of segmentally duplicated genes. As shown in Additional file 3: Table S3, we found that a tandem duplicated gene pair already existed before the segmental duplication event occurred in the formation of the StnsLTP gene family. Gene duplication is essential in the expansion and functional diversification of the gene family [64]. This is very similar to our research. Our analysis showed that duplication events not only contribute to the amplification of the StnsLTP genes family but also cause genetic diversity in the evolutionary process. Previous studies have shown that duplicated nsLTP genes have similar expression patterns.

Gene expression patterns found by RNA-seq of nsLTP genes in different organs and/or tissues have been reported in a large number of species, such as Arabidopsis, rice, peppers, and tomatoes. We used the available potato RNA-seq data [33] to analyze the expression pattern profiles of nsLTPs in different tissues/organs to elucidate their functional roles. From the heat map (Fig. 6), the expression levels of most StnsLTP genes in various tissues/organs are significantly different, which suggests that there are differences in the functions of different StnsLTPs. Among these genes, StnsLTPII.2, StnsLTPIV.3, StnsLTPVIII.1, StnsLTPVIII.9, StnsLTPVIII.11, and StnsLTPXIII.8 were highly expressed in the storage organs of both DM and RH (Fig. 6). Compared with the results of RNA-seq, the results of qRT-PCR showed that StnsLTPII.2, StnsLTPVIII.9, and StnsLTPXIII.8 were highly expressed in stolons and/or young tubers (Fig. 7), indicating that these genes may be involved in tuberization and/or tuber development. Many studies have shown that nsLTPs are involved in sexual reproduction processes such as pollen development, pollen wall formation and fertilization [65, 66]. We found that StnsLTPI.2, StnsLTPI.3, StnsLTPI.4, StnsLTPI.5, StnsLTPI.29, StnsLTPI.36, StnsLTPIV.10, and StnsLTPXIII.3 are highly expressed in flowers, indicating that these genes may be involved in flower development process. This is very similar to the results of qRT-PCR. However, the expression profiles generated by qRT-PCR are not always consistent with the expression pattern represented by the RNA-seq data. For example, for StnsLTPVIII.1, in the RNA-seq data, the part with the highest expression is the floral organ, but the result of qRT-PCR result shows the stolon. There may be many reasons for such discrepancies. The potato RNA-seq data used in our study are expressed as FPKM values. FPKM values may be affected by highly expressed genes [67] Therefore, the deviation of the FPKM value results in a difference from the result of qRT-PCR. For the potato, which is the fourth largest food crop in the world, we are 
most concerned about the development of tubers. The tubers of potato are metamorphic organs formed by swelling of the tops of the underground stolons. Due to the rapid increase in starch content at the top of the stolon and the sharp reduction in reducing sugar content at the initial stage of tuber formation, Hawker et al. suggested that starch synthesis may be necessary for tuber development [68]. It is now generally accepted that the formation of tubers is caused by an increase in the number and size of cells in outer medulla, cortex, and inner medulla [69]. In previous studies, xylogen-like arabinogalactan protein was found to be an arabinogalactan protein with an nsLTP domain that can boost xylem cell formation [70]. In a previous study, it was found that the expression of some members of the StnsLTP family is highly tissue-specific and occurs at a critical stage in the life cycle of potato tubers [71]. We also found that some genes are highly expressed in potato tubers, such as StnsLTPXIII.8. Therefore, they may be xylogen-arabinogalactan proteins that are involved in xylem formation in potato tubers.

The pattern of gene expression is also affected by the promoter region, which contains transcription factor binding sites. The number and type of cis-acting elements involved in a promoter are very important for gene expression under various stresses, including light, heat, drought, and wounding, and hormone signaling (e.g., ethylene, gibberellin, cytokinin, auxin). Plant hormones are trace organic substances that are synthesized in plants and have significant effects on plant growth. Therefore, understanding the type and number of cis-acting elements related to hormones involved in the promoter is of great significance for the future studies of the StnsLTP gene family in regulating the molecular mechanisms of potato tuber development. Among the many hormones associated with plant growth, GA3 and ABA are recognized as the most relevant factors for tuber formation. GA3 inhibits tuber formation, while ABA promotes tuber formation [72]. Moreover, several studies have shown that $n s L T P$ genes can respond to certain signaling molecules, such as abscisic acid, salicylic acid and ethylene [73-76]. In addition, we found that the promoters of the StnsLTP gene family contain a wide variety of cis-acting elements associated with hormonal responses, such as the GARE motif and CGTCA motif. This indicates that some members of the StnsLTP gene family respond to hormonal signaling molecules during potato growth and development. For example, StnsLTPI.29 contains the most cis-acting elements that respond to hormones. Previous studies have shown that different nsLTP gene expression can produce different responses to abiotic stress [77]. The expression of nsLTP genes is usually induced by environmental factors such as low temperature [73, 74, 78, 79], drought [74, 80], and high temperature [74, 80]. In Arabidopsis, AtLTPg4 plays an important role in wax and/or keratin monomer transport [81]. AtLTP2 is involved in the integrity of the cell wall of the stratum corneum [22], and AtLTP3 responds to freezing and drought stress signals [24]. To date, the most in-depth study of nsLTPs in potato has examined the StnsLTP.1 protein [29], which can respond to a variety of abiotic stresses, including the main environmental factors that limit potato production - heat, drought, and salinity [34]. In addition, we analyzed its cis-acting elements and discovered that they include not only cis-acting elements that respond to abiotic stress, such as HSE, and TC-rich repeats but also other functional cis-acting elements, such as ABRE, that respond to abscisic acid.

\section{Conclusion}

The nsLTP family is found in a variety of plant species and participates in a variety of developmental and physiological processes. However, there has been a lack of information about this family in the potato genome. In this study, we performed a genome-wide analysis of the nsLTP family in potato and identified 83 StnsLTP genes. This study predicted and analyzed the different biochemical characteristics of StnsLTP proteins. Based on sequence similarity in the ECM region, StnsLTP proteins are divided into 8 types (I, II, IV, V, VII, VIII, XII, and XIII). Subsequently, a variety of bioinformatics methods and qRT-PCR methods were used to analyze gene structure, phylogeny, chromosomal location, gene duplication, and KaKs ratios, cis-acting elements associated with growth and development, GO annotation and expression patterns in different tissues. Furthermore, the StnsLTP genes in potato identified by us provide not only the basis for functional research into the StnsLTP genes but also a valuable reference for further study of the biological functions of the gene family and its related pathways and mechanisms.

\section{Additional files}

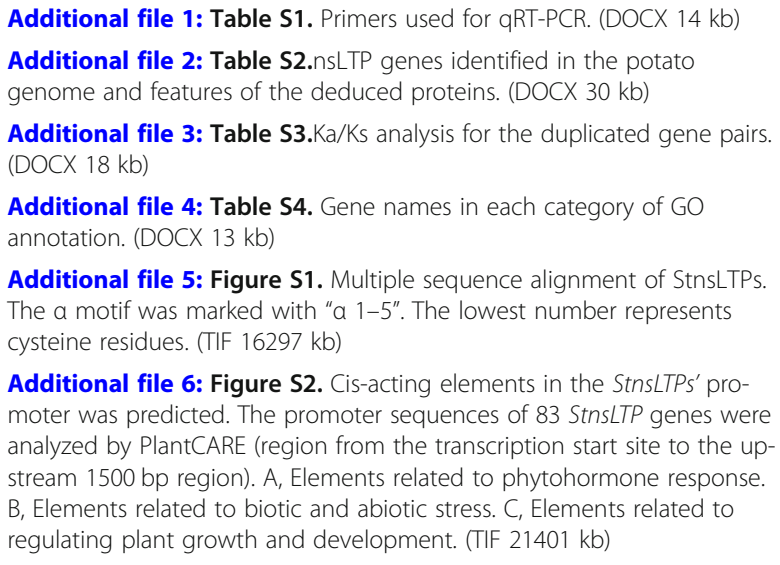

Additional file 5: Figure S1. Multiple sequence alignment of StnsLTPS. The a motif was marked with "a 1-5". The lowest number represents cysteine residues. (TIF $16297 \mathrm{~kb}$ )

Additional file 6: Figure S2. Cis-acting elements in the StnsLTPs' promoter was predicted. The promoter sequences of 83 StnsLTP genes were analyzed by PlantCARE (region from the transcription start site to the upstream 1500 bp region). A, Elements related to phytohormone response. B, Elements related to biotic and abiotic stress. C, Elements related to regulating plant growth and development. (TIF $21401 \mathrm{~kb}$ ) 


\section{Abbreviations}

AA: Amino acid; BLASTp: Basic local alignment search tool-protein; HMM: Hidden markov model; ORF: Open reading frame; PGDD: Plant genome duplication database; PGSC: Potato genome sequencing consortium; QRT-PCR: Quantitative real-time polymerase chain reaction; tBLASTn: Protein to translated nucleotide BLAST

\section{Acknowledgements}

We thank the members of Dr. Chen's lab in Northwest Agriculture \& Forestry University for providing technical laboratory advice.

\section{Funding}

This research was supported by the National Natural Science Foundation of China (31500159). The funder had no role in study design, data collection and analysis, decision to publish, or preparation of the manuscript.

\section{Availability of data and materials}

All of the datasets supporting the results of this article are included within the article and its Additional files. The raw data generated in this study can be obtained by reasonable request to the corresponding author.

\section{Authors' contributions}

$H M$ and $G L$ conceived and designed the research plans; GL, MH, and $Y L$ performed most of the experiments and analyzed the data; YP, MY, CH, YZ (Yaqi Zhao) and YZ (Yao Zhou) provided technical assistance to GL, MH, and $Y L ; G L$ wrote the article with contributions of all the authors; HM supervised and complemented the writing. All of the authors have read and approved the final manuscript

\section{Ethics approval and consent to participate}

Not applicable.

\section{Consent for publication}

Not applicable.

\section{Competing interests}

The authors declare that they have no competing interests.

\section{Publisher's Note}

Springer Nature remains neutral with regard to jurisdictional claims in published maps and institutional affiliations.

\section{Author details}

${ }^{1}$ College of Agronomy, Northwest A\&F University, Yangling 712100, Shaanxi, China. ${ }^{2}$ Innovation Experimental College, Northwest A\&F University, Yangling 712100, Shaanxi, China.

Received: 2 January 2019 Accepted: 15 April 2019

Published online: 14 May 2019

\section{References}

1. Kader JC, Julienne M, Vergnolle C. Purification and characterization of a spinach-leaf protein capable of transferring phospholipids from liposomes to mitochondria or chloroplasts. Eur J Biochem. 1984;139(2):411-6.

2. Silva PD, Landon C, Industri B, Marais A, Marion D, Ponchet M, Vovelle F. Solution structure of a tobacco lipid transfer protein exhibiting new biophysical and biological features. Proteins. 2005:59(2):356-67.

3. Salminen TA, Blomqvist K, Edqvist J. Lipid transfer proteins: classification, nomenclature, structure, and function. Planta. 2016;244(5):971-97.

4. Boutrot F, Chantret N, Gautier MF. Genome-wide analysis of the rice and arabidopsis non-specific lipid transfer protein (nsLtp) gene families and identification of wheat nsLtp genes by EST data mining. BMC Genomics 2008;9(1):86

5. Liu F, Zhang XB, Lu CM, Zeng XH, Li YJ, Fu DH, Wu G. Non-specific lipid transfer proteins in plants: presenting new advances and an integrated functional analysis. J Exp Bot. 2015;66(19):5663-81.

6. Liu W, Huang D, Kan L, Hu S, Yu J, Gang G, Song S. Discovery, identification and comparative analysis of non-specific lipid transfer protein (nsLtp) family in Solanaceae. Genomics Proteomics Bioinformatics. 2010;08(4):229-37.
7. Li J, Gao G, Xu K, Chen B, Yan G, Li F, Qiao J, Zhang T, Wu X. Genome-wide survey and expression analysis of the putative non-specific lipid transfer proteins in Brassica rapa L. PLoS One. 2014;9(1):e84556.

8. Edstam MM, Viitanen L, Salminen TA, Edqvist J. Evolutionary history of the non-specific lipid transfer proteins. Mol Plant. 2011;4(6):947-64.

9. Yeats TH, Rose JKC. The biochemistry and biology of extracellular plant lipid-transfer proteins (LTPS). Protein Sci. 2008;17(2):191-8.

10. Wei KF, Zhong XJ. Non-specific lipid transfer proteins in maize. BMC Plant Biol. 2014;14(1):281.

11. Carvalho AD, Gomes VM. Role of plant lipid transfer proteins in plant cell physiology - a concise review. Peptides. 2007;28(5):1144-53.

12. Li F, Fan K, Ma FL, Yue EK, Bibi N, Wang M, Shen H, Hasan MMU, Wang XD. Genomic identification and comparative expansion analysis of the non-specific lipid transfer protein gene family in Gossypium. Sci Rep. 2016;6(1):38948.

13. Garcíaolmedo F, Molina A, Segura A, Moreno M. The defensive role of nonspecific lipid-transfer proteins in plants. Trends Microbiol. 1995;3(2):72.

14. Cameron KD, Teece MA, Smart LB. Increased accumulation of Cuticular wax and expression of lipid transfer protein in response to periodic drying events in leaves of tree tobacco. Plant Physiol. 2006;140(1):176-83.

15. Yeats TH, Rose JKC. The formation and function of plant cuticles. Plant Physiol. 2013;163(1):5.

16. Schreiber L. Transport barriers made of cutin, suberin and associated waxes. Trends Plant Sci. 2010;15(10):546-53.

17. Kunst L, Samuels AL. Biosynthesis and secretion of plant cuticular wax. Prog Lipid Res. 2003:42(1):51-80.

18. Maldonado AM, Doerner P, Dixon RA, Lamb CJ, Cameron RK. A putative lipid transfer protein involved in systemic resistance signalling in Arabidopsis. Nature. 2002;419(6905):399-403.

19. Lascombe MB, Bakan B, Buhot N, Marion D, Blein JP, Larue V, Lamb C, Prangé T. The structure of \&ldquo;defective in induced resistance\&rdquo; protein of Arabidopsis thaliana, DIR1, reveals a new type of lipid transfer protein. Protein Sci. 2008;17(9):1522-30.

20. Sels J, Mathys J, De BC, Cammue BP, De MB. Plant pathogenesis-related (PR) proteins: a focus on PR peptides. Plant Physiol Biochem. 2008:46(11):941-50.

21. Wang SY, Wu J, Ye XY, Rao PF. A non-specific lipid transfer protein with antifungal and antibacterial activities from the mung bean. Peptides. 2004;25(8):1235.

22. Jacq A, Pernot C, Martinez Y, Domergue F, Payre B, Jamet E, Burlat V, Pacquit VB. The Arabidopsis lipid transfer protein 2 (AtLTP2) is involved in cuticle-Cell Wall Interface integrity and in etiolated hypocotyl permeability. Front Plant Sci. 2017:8:263.

23. Shin D, Moon SJ, Han S, Kim BG, Sang RP, Lee SK, Yoon HJ, Lee HE, Kwon HB, Baek D. Expression of StMYB1R-1, a novel potato single MYB-like domain transcription factor, increases drought tolerance. Plant Physiol. 2011; 155(1):421-32.

24. Guo L, Yang HB, Zhang XY, Yang SH. Lipid transfer protein 3 as a target of MYB96 mediates freezing and drought stress in Arabidopsis. J Exp Bot. 2013; 64(6):1755-67.

25. Zou HW, Tian XH, Ma GH, Li ZX. Isolation and functional analysis ofZmLTP3, a homologue toArabidopsis LTP3. Int J Mol Sci. 2013;14(3):5025.

26. Kiba A, Nakatsuka T, Yamamura S, Nishihara M. Gentian lipid transfer protein homolog with antimicrobial properties confers resistance to Botrytis cinerea in transgenic tobacco. Plant Biotechnology. 2012;29(1):95-101.

27. Lee SB, Go YS, Bae HJ, Park JH, Cho SH, Cho HJ, Lee DS, Park OK, Hwang I, Suh MC. Disruption of glycosylphosphatidylinositol-anchored lipid transfer protein gene altered cuticular lipid composition, increased plastoglobules, and enhanced susceptibility to infection by the fungal pathogen Alternaria brassicicola. Plant Physiol. 2009;150(1):42.

28. Gang G, Jin LP, Xie KY, Qu DY. The potato StLTPa7 gene displays a complex Ca2+-associated pattern of expression during the early stage of potatoRalstonia solanacearum interaction. Mol Plant Pathol. 2009;10(1):15-27.

29. Gangadhar BH, Kappachery S, Jelli V, Venkidasamy B, Kumar A, Yu JW, Ram P, Mishra RK. Enhanced Tolerance of Transgenic Potato Plants OverExpressing Non-specific Lipid Transfer Protein-1 (StnsLTP1) against Multiple Abiotic Stresses. Front Plant Sci. 2016:7:1228.

30. Tapia G, Morales-Quintana L, Parra C, Berbel A, Alcorta M. Study of nsLTPs in Lotus japonicus genome reveal a specific epidermal cell member (LjLTP10) regulated by drought stress in aerial organs with a putative role in cutin formation. Plant Mol Biol. 2013:82(4-5):485-501.

31. Wang NJ, Lee CC, Cheng CS, Lo WC, Yang YF, Chen MN, Lyu PC. Construction and analysis of a plant non-specific lipid transfer protein database (nsLTPDB). BMC Genomics. 2012;13(1):S9. 
32. D'Agostino N, Buonanno M, Ayoub J, Barone A, Monti SM, Rigano MM. Identification of non-specific lipid transfer protein gene family members in Solanum lycopersicum and insights into the features of sola 13 protein. Sci Rep. 2019;9(1):1607.

33. Xu X, Pan SK, Cheng SF, Zhang B, Mu DS, Ni PX, Zhang GY, Yang S, Li RQ, Wang J, et al. Genome sequence and analysis of the tuber crop potato. Nature. 2011;475(7355):189-U194.

34. Finn RD, Clements J, Eddy SR. HMMER web server: interactive sequence similarity searching. Nucleic Acids Res. 2011;39:W29-37.

35. Guerche P, Tire C, Sa FGD, Clercq AD, Montagu MV, Krebbers E. Differential Expression of the Arabidopsis $2 S$ Albumin Genes and the Effect of Increasing Gene Family Size. Plant Cell. 1990;2(5):469.

36. Strobl S, Maskos K, Wiegand G, Huber R, Gomisrüth FX, Glockshuber R. A novel strategy for inhibition of alpha-amylases: yellow meal worm alphaamylase in complex with the Ragi bifunctional inhibitor at $2.5 \mathrm{~A}$ resolution. Structure. 1998;6(7):911.

37. Kumar S, Stecher G, Tamura K. MEGA7: Molecular Evolutionary Genetics Analysis Version 7.0 for Bigger Datasets. Mol Biol Evol. 2016;33(7):1870.

38. Wang LQ, Guo K, Li Y, Tu YY, Hu HZ, Wang BR, Cui XC, Peng LC. Expression profiling and integrative analysis of the CESA/CSL superfamily in rice. BMC Plant Biol. 2010;10(1):282.

39. Wang D, Zhang Y, Zhang Z, Jiang Z, Yu J. KaKs_Calculator 2.0:a toolkit incorporating gamma-series methods and sliding window strategies. Genomics, Proteomics Bioinformatics. 2010;8(1):77-80

40. Lescot M, Dehais P, Thijs G, Marchal K, Moreau Y, Van de Peer Y, Rouze P, Rombauts S. PlantCARE, a database of plant cis-acting regulatory elements and a portal to tools for in silico analysis of promoter sequences. Nucleic Acids Res. 2002;30(1):325-7.

41. Götz S, Garcíagómez JM, Terol J, Williams TD, Nagaraj SH, Nueda MJ, Robles M, Talón M, Dopazo J, Conesa A. High-throughput functional annotation and data mining with the Blast2GO suite. Nucleic Acids Res. 2008;36(10):3420-35.

42. Edstam M. M, Viitanen, Lenita, Salminen, Tiina a, Edqvist, Johan: evolutionary history of the non-specific lipid transfer proteins. Mol Plant. 2011;04(6):947-64.

43. Kloosterman B, Visser RG, Bachem CW. Isolation and characterization of a novel potato auxin/Indole-3-acetic acid family member (StIAA2) that is involved in petiole hyponasty and shoot morphogenesis. Plant Physiol Biochem. 2006:44(11-12):766-75.

44. Wang RQ, Zhao P, Kong NN, Lu RZ, Pei Y, Huang CX, Ma HL, Chen Q. Genome-Wide Identification and Characterization of the Potato bHLH Transcription Factor Family. Genes-Basel. 2018;9(1):54.

45. Nicot N, Hausman JF, Hoffmann L, Evers D. Housekeeping gene selection for real-time RT-PCR normalization in potato during biotic and abiotic stress. J Exp Bot. 2005;56(421):2907-14.

46. Ma HL, Zhao J. Genome-wide identification, classification, and expression analysis of the arabinogalactan protein gene family in rice (Oryza sativa L.). J Exp Bot. 2010;61(10):2647-68.

47. Cannon SB, Mitra A, Baumgarten A, Young ND, May G. The roles of segmental and tandem gene duplication in the evolution of large gene families in Arabidopsis thaliana. BMC Plant Biol. 2004;4(1):10.

48. Wei KF, Chen YH, Xie DX. Genome-scale evolution and phylodynamics of H5N1 influenza virus in China during 1996-2012. Vet Microbiol. 2013;167(3-4): 383-93.

49. Yang ZH, Bielawski JP. Statistical methods for detecting molecular adaptation. Trends Ecol Evol. 2000;15(12):496-503.

50. Xu G, Guo C, Shan H, Kong H. Divergence of duplicate genes in exon-intron structure. Proc Natl Acad Sci U S A. 2012;109(4):1187-92.

51. Yoshida K, Schuenemann VJ, Cano LM, Pais M, Mishra B, Sharma R, Lanz C, Martin FN, Kamoun S, Krause J. Correction: the rise and fall of the Phytophthora infestans lineage that triggered the Irish potato famine. Elife. 2013;2(2):e00731.

52. Levy D, Veilleux RE. Adaptation of potato to high temperatures and salinity - a review. Am J Potato Res. 2007;84(6):487-506.

53. Martín-Pedraza L, González M, Gómez F, Blanca-Gómez N, Arandia MG, Rodríguez R, Torres MJ, Blanca M, Villalba M, Mayorga C. Two non-specific lipid transfer proteins (nsLTP) from tomato seeds are associated to severe symptoms of tomato-allergic patients. Mol Nutr Food Res. 2015;60(5):1172-82.

54. Bun NT, Fai CRC, Ho WJ, Ye X. Lipid-transfer proteins. Pept Sci. 2012;98:268-79.

55. Loon CDV. The effect of water stress on potato growth, development, and yield. American Potato Journal. 1981;58(1):51-69.

56. Moore RC, Purugganan MD. The evolutionary dynamics of plant duplicate genes. Curr Opin Plant Biol. 2005;8(2):122-8.
57. Flagel LE, Wendel JF. Gene duplication and evolutionary novelty in plants. New Phytol. 2009;183(3):557-64.

58. Mattick JS, Gagen MJ. The evolution of controlled multitasked gene networks: the role of introns and other noncoding RNAs in the development of complex organisms. Mol Biol Evol. 2001;18(9):1611.

59. Ren XY, Vorst O, Fiers MWEJ, Stiekema WJ, Nap JP. In plants, highly expressed genes are the least compact. Trends Genet. 2006;22(10):528-32.

60. Jeffares D. Rapidly regulated genes are intron poor. Trends Genet. 2008; 24(10):488.

61. Vision TJ, Brown DG, Tanksley SD. The origins of genomic duplications in Arabidopsis. Science. 2000;290(5499):2114-7.

62. Kong H, Landherr LL, Frohlich MW, Leebens-Mack J, Ma H, Depamphilis CW. Patterns of gene duplication in the plant SKP1 gene family in angiosperms: evidence for multiple mechanisms of rapid gene birth. Plant J. 2007;50(5):873-85.

63. Kaul S, Koo HL, Jenkins J, Rizzo M, Rooney T, Tallon LJ, Feldblyum T, Nierman W, Benito MI, Lin XY, et al. Analysis of the genome sequence of the flowering plant Arabidopsis thaliana. Nature. 2000;408(6814):796-815.

64. Lavin M, Herendeen PS, Wojciechowski MF. Evolutionary rates analysis of Leguminosae implicates a rapid diversification of lineages during the tertiary. Syst Biol. 2005;54(4):575-94

65. Edstam MM, Edqvist J. Involvement of GPI-anchored lipid transfer proteins in the development of seed coats and pollen in Arabidopsis thaliana. Physiol Plant. 2014;152(1):32-42.

66. Huang MD, Chen TLL, Huang AHC. Abundant type III lipid transfer proteins in Arabidopsis Tapetum are secreted to the Locule and become a constituent of the pollen Exine. Plant Physiol. 2013:163(3):1218-29.

67. Kukurba KR, Montgomery SB. RNA sequencing and analysis. Cold Spring Harb Protoc. 2015;2015(11):951.

68. Hawker JS, Marschner H, Krauss A. Starch synthesis in developing potato tubers. Physiol Plant. 1979;46(1):25-30.

69. Kolomiets MV, Gladon RJ. Lipoxygenase is involved in the control of potato tuber development. Plant Cell. 2001;13(3):613-26.

70. Kobayashi $Y$, Motose H, Iwamoto K, Fukuda H. Expression and genome-wide analysis of the Xylogen-type gene family. Plant Cell Physiol. 2011;52(6): 1095-106.

71. Horvath BM, Bachem CWB, Trindade LM, Oortwijn MEP, Visser RGF. Expression analysis of a family of nsLTP genes tissue specifically expressed throughout the plant and during potato tuber life cycle. Plant Physiol. 2002; 129(4):1494-506.

72. Lovell PH, Booth A. Effects of GA on growth, tuber formation and carbohydrate distribution in Solanum tuberosum. New Phytol. 1967;66(4):525-37.

73. Yuberoserrano EM, Moyano E, Medinaescobar N, Muñozblanco J, Caballero JL. Identification of a strawberry gene encoding a non-specific lipid transfer protein that responds to ABA, wounding and cold stress. J Exp Bot. 2003;54(389):1865-77.

74. Wu GH, Robertson AJ, Liu XJ, Zheng P, Wilen RW, Nesbitt NT, Gusta LV. A lipid transfer protein gene BG-14 is differentially regulated by abiotic stress, $\mathrm{ABA}$, anisomycin, and sphingosine in bromegrass (Bromus inermis). J Plant Physiol. 2004;161(4):449-58.

75. Federico ML, Kaeppler HF, Skadsen RW. The complex developmental expression of a novel stress-responsive barley Ltp gene is determined by a shortened promoter sequence. Plant Mol Biol. 2005;57(1):35-51.

76. Maghuly F, Borrotofernandez EG, Khan MA, Herndl A, Marzban G, Laimer M. Expression of calmodulin and lipid transfer protein genes in Prunus incisa $\times$ serrula under different stress conditions. Tree Physiol. 2009;29(3):437-44.

77. Jang C, Kim D, Bu S, Kim J, Lee S, Kim J, Johnson J, Seo Y. Isolation and characterization of lipid transfer protein (LTP) genes from a wheat-rye translocation line. Plant Cell Rep. 2002;20(10):961-6.

78. Gaudet DA, Laroche A, Frick MR, Puchalski B. Cold induced expression of plant defensin and lipid transfer protein transcripts in winter wheat. Physiol Plant. 2003;117(2):195-205.

79. Hwang EW, Kim KA, Park SC, Jeong MJ, Byun MO, Kwon HB. Expression profiles of hot pepper ( capsicum annuum ) genes under cold stress conditions. J Biosci. 2005;30(5):657-67.

80. Gonorazky AG, Regente MC. De ICL: stress induction and antimicrobial properties of a lipid transfer protein in germinating sunflower seeds. J Plant Physiol. 2005;162(6):618

81. Kim H, Lee SB, Kim HJ, Min MK, Hwang I, Suh MC. Characterization of glycosylphosphatidylinositol-anchored lipid transfer protein 2 (LTPG2) and overlapping function between LTPG/LTPG1 and LTPG2 in cuticular wax export or accumulation in Arabidopsis thaliana. Plant Cell Physiol. 2012; 53(8):1391. 